\title{
Development and characterization of drug-loaded biodegradable PLA microcarriers prepared by the electrospraying technique
}

\author{
JIAN LU, REN HOU, ZHENLEI YANG and ZHIHONG TANG \\ Department of Orthopedics, Affiliated Hospital of Guilin Medical University, Guilin, Guangxi 541001, P.R. China
}

Received September 3, 2014; Accepted April 17, 2015

DOI: $10.3892 / \mathrm{ijmm} .2015 .2201$

\begin{abstract}
Biodegradable particles are extremely useful in the development of novel drug delivery systems. Recent studies have suggested that morphology can influence the mechanisms of drug delivery in many ways. In the present study, biodegradable microparticles with different morphologies were prepared from poly(L-lactide) (PLA) using the electrospraying technique. The microparticles were then systematically examined by scanning using an electron microscope. The results revealed that the preparation of drug-loaded microspheres through electrospraying is a simple and efficient method, and the processing parameters, such as polymer molecular weight, concentration, surfactant and solvent play an important role in obtaining high quality microcarriers. The association between microcarrier morphology and the processing parameters used was also investigated. Rifampin-loaded PLA microspheres were also prepared according to the above-mentioned model. Our data demonstrate that the drug release from PLA microspheres can be sustained in vitro for over $60 \mathrm{~h}$. Our study focused on obtaining electrosprayed medicated microparticles from complex polyester particles. Further studies are required to explore the potential commercial use of these microparticles.
\end{abstract}

\section{Introduction}

Biodegradable micro/nanoparticles are extremely useful in the development of novel drug delivery systems. They can carry densely loaded drug solutions, improve drug stability and bioavailability, and extend the effects of drugs or genes through sustained release. Besides, they provide a versatile platform for the delivery of various types of compounds, ranging from small molecules to macromolecules, such as proteins and oligonucleotides. Synthetic particles of diverse forms have gained increasing attention, as evident from recent studies, which suggested that morphology can influence the mechanisms of drug delivery in many ways (1-4). For example, an optimum

Correspondence to: Dr Zhihong Tang, Department of Orthopedics, Affiliated Hospital of Guilin Medical University, 15 Lequn Road, Guilin, Guangxi 541001, P.R. China

E-mail: tangzhihong313@sina.com

Key words: electrospray, poly(L-lactide), morphology, drug delivery form or shape can help tailor the kinetics of the drug release, and even avoid the initial burst (5). The morphology of polymer particles also highly influences their transport and circulation inside the body $(6,7)$.

The aliphatic polyesters, specifically poly(L-lactide) (PLA), polyglycolide (PGA) and their copolymer, poly(lactide-coglycolide) (PLGA) $(8,9)$ are some of the most widely used materials in medical treatment, due to their superior biodegradability and biocompatibility. Over the past decade, considerable progress has been made in the design of these drug carriers, which includes the modification of the particle surface to improve stability and circulation throughout the body (10), biospecific targeting of cellular ligands or extracellular matrix components (11-14) and the incorporation of diagnostic imaging agents (15).

To date, a variety of techniques have been used to produce polymeric particles, such as suspension (16) or emulsion (17) polymerization, solvent evaporation (18) spray drying (19-21) and the Shirasu porous glass (SPG) membrane emulsification technique (22). However, the majority of these methods cannot be used to synthesize particles with varying morphologies. Gel microparticles have also been generated by microfluidic devices (23). The use of various electrical potential differences for droplet formation is known as electrospraying, wherein the formation depends on the actual jet breakup wavelength under flow instabilities (24). A number of polymer particles, such as poly(methyl methacrylate) (PMMA) (25), polyvinylidine fluoride (PVDF) (26), polystyrene (PS) (27) have been produced using electrospray technology; however, to date, few studies on PLA particles with different morphologies prepared by electrospraying for drug loading are available in the literature.

In this study, we not only synthesized PLA microparticles by electrospray technology, but also loaded rifampin into the PLA microcarrier using the electrospraying technique and investigated the release behavior in vitro.

\section{Materials and methods}

Materials. The PLA microparticles were synthesized in our laboratory. The molecular weights were determined by gel permeation chromatography (GPC). The results are presented in Table I. Rifampin, proteinase $\mathrm{K}$ and triethyl benzyl ammonium chloride (TEBAC, $\mathrm{C}_{13} \mathrm{H}_{22} \mathrm{CIN}$ ) were obtained commercially. Tris(hydroxymethyl)aminomethane (Tris-base) was supplied by Merck and used without further purification to prepare the 


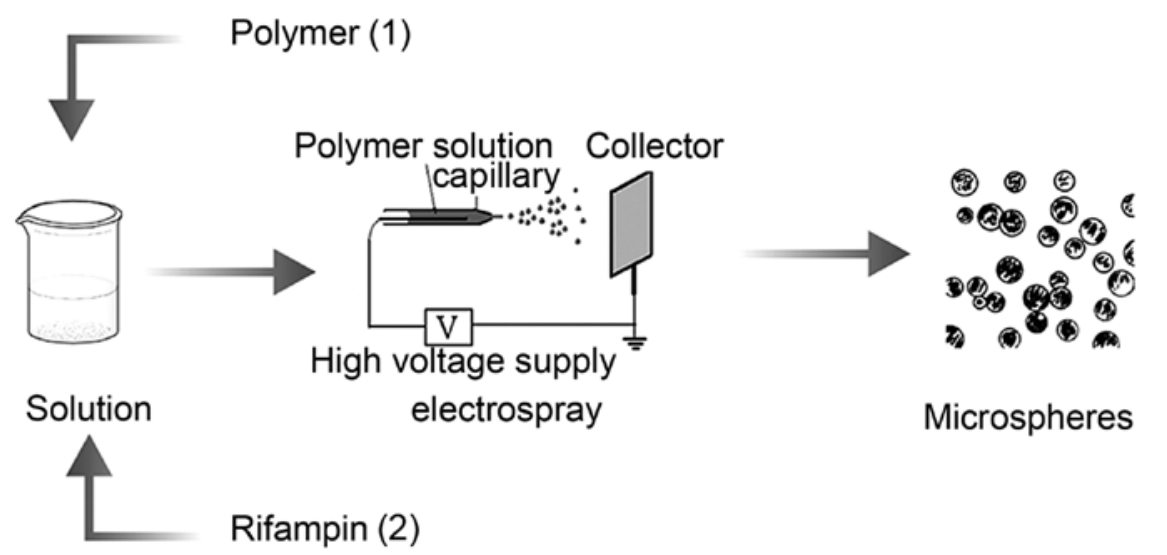

Figure 1. Experimental unit for electrospraying. Preparation of polymer microshperes loaded with rifampin. PLA $\left(\mathrm{Mn}=6.29 \mathrm{x} 10^{3}\right)$ was dissolved in chloroform to prepare a $10 \%(\mathrm{w} / \mathrm{v})$ solution. Subsequently, $3 \%$ TEBAC and 3\% of rifampin were added to the polymer solution. After $24 \mathrm{~h}$, the solution was electrosprayed. PLA, poly(L-lactide); Mn, number average molecular weight; TEBAC, triethyl benzyl ammonium chloride.

Tris- $\mathrm{HCl}$ buffer solution of $\mathrm{pH}$ 8.6. Chloroform and acetone were of analytical grade.

Polymer processing by electrospray technology. In the preparation of precursor solutions, chloroform was used as the solvent for PLA. Generally, a controlled amount of PLA is dissolved in chloroform, and then $5 \%$ of TEBAC (w/v) with respect to the polymer used was added to the polymer solution, along with continuous magnetic stirring at room temperature for $24 \mathrm{~h}$. Consequently, PLA solutions of different molecular weights were obtained.

The polymer solution was transferred to a 5-ml syringe attached to a stainless steel blunt nozzle with an internal diameter of $0.4 \mathrm{~mm}$, which was connected to a high-voltage power supply (Acopian Technical Co., Easton, PA, USA). The highvoltage power supply was used to generate a $5 \mathrm{kV}$ potential difference between the nozzle and the grounded aluminum foil. The distance used for spraying the PLA solution was adjustable. A schematic diagram of the experiment is presented in Fig. 1.

Characteristics of the polymer products. A scanning electron microscope (SEM, JSM 5600LV) was used to observe the morphology of the gold-coated polymer products at a $20 \mathrm{kV}$ acceleration voltage. Fourier transform infrared(FTIR; Bio-Rad Win-IR) spectroscopic analysis of the electrosprayed polymer products was carried out over a range of $400-4,000 \mathrm{~cm}^{-1}$ at a resolution of $2 \mathrm{~cm}^{-1}$.

In vitro release of the drug. The polymer microsphere solutions containing a specific amount of the drug were sealed in a dialysis bag (molecular weight cut-off, 30,000) and incubated at $37^{\circ} \mathrm{C}$ in $200 \mathrm{ml}$ of Tris- $\mathrm{HCl}$ buffer solution (pH 8.6, $0.05 \mathrm{M}$ ) containing $3 \mu \mathrm{g} / \mathrm{ml}$ of proteinase $\mathrm{K}$. Proteinase $\mathrm{K}$ was not added for the blank control. At pre-determined time intervals, $4 \mathrm{ml}$ of the medium was released and replaced with an equal quantity of buffer. The concentration of the drug in the release medium was determined directly using a UV-Visible spectrophotometer (WFZ UV-2102; Shanghai Unique Machinery Technology Co., Ltd, Shanghai, China) at $473 \mathrm{~nm}$. The concentration of rifampin in the release medium was confirmed by comparing the UV absorbance of rifampin with a standard calibration curve of rifampin in the same buffer. Thereafter, the total amount of rifampin released was calculated as a function of incubation time.

Stability test. The stability of the microspheres loaded with rifampin was also tested. Two groups of samples were exposed to air for 140 days. The samples were collected on day 70 and day 140 and analyzed for their appearance, drug concentration and release percentage.

\section{Results}

Effect of different parameters on the morphology of the PLA microparticles. The concentration used influences the morphology of the electrospray products. SEM images of the products dissolved in chloroform at various concentrations of PLA $\left(\mathrm{Mn}=5.62 \times 10^{4}\right)$ are shown in Fig. 2. Only microfibers or nanofibers were formed using a $5 \%(\mathrm{w} / \mathrm{v})$ PLA solution (Fig. 2A). By decreasing the PLA concentration to 2-3\% of its original molecular weight, a beads-on-a-string structure was observed (Fig. 2B and C). Further dilution resulted in a decrease in the quantity and the diameter of fiber, while the number of beads increased, and the shape of the beads changed to a semispherical shape (Fig. 2D). The fibers disappeared and only hollow semi-shperical particles were present at a concentration equal or $<1 \%$ weight (Fig. $2 \mathrm{E}$ and F).

The effect of polymeric molecular weights on the morphology of the resulting products is shown in Fig. 3. In the case of a high molecular weight, the morphology of the electrospray particles assumed a bowel-like structure (Fig. 3A); with the decreasing molecular weight, the bowel wall thickened. A spherical transformation occurred with a reduction in the polymer molecular weight, with dents in the spherical structure (Fig. 3B). When the polymer molecular weight was decreased to a lesser extent, a smooth microsphere was formed (Fig. 3C).

The effect of surfactant on the morphology of the products is shown in Fig. 4. The products with TEBAC assumed a hollow semi-spherical structure, and their surfaces were compact and smooth (Fig. 4B), while those without TEBAC presented a cake-like structure with few of pores on their surface (Fig. 4A). 


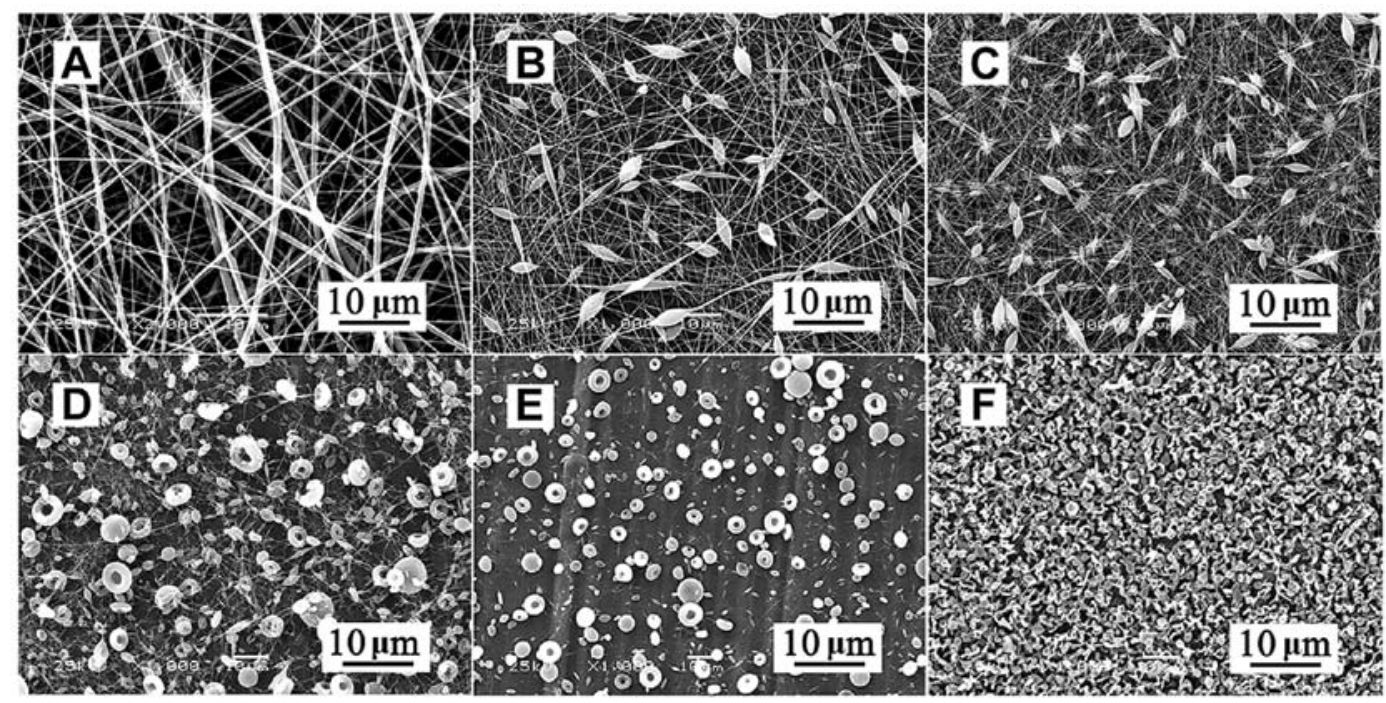

Figure 2. SEM images of electrosprayed PLA $\left(\mathrm{Mn}=5.62 \times 10^{4}\right) / \mathrm{CHCl}_{3}$ solutions at various concentrations: (A) $5 \%$, (B) $3 \%$, (C) $2 \%$, (D) $1.5 \%$, (E) $1 \%$, and (F) $0.5 \%$. TEBAC (5\%) was added to the above polymer solutions. SEM, scanning electron microscopy; PLA, poly(L-lactide); Mn, number average molecular weight; TEBAC, triethyl benzyl ammonium chloride.

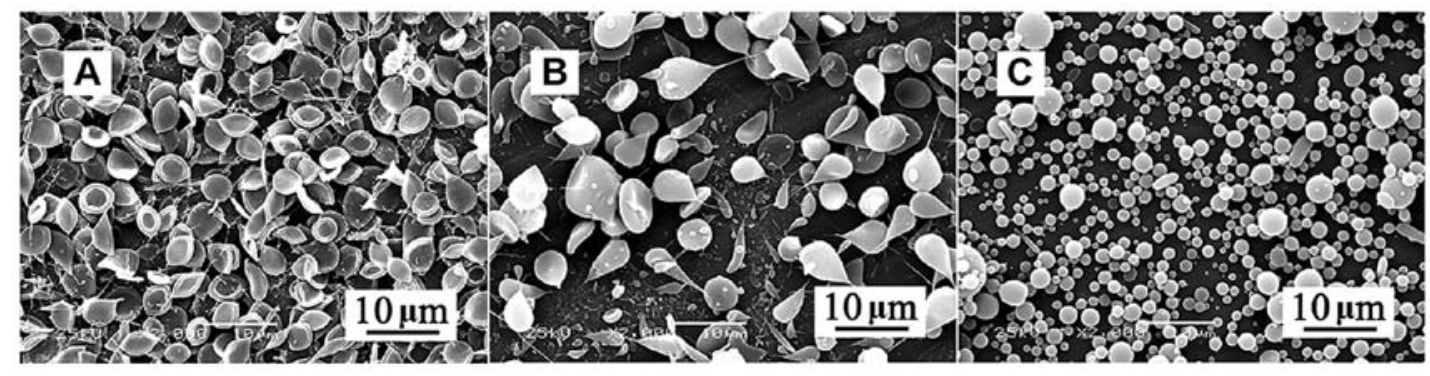

Figure 3. SEM images of electrospraying different $\mathrm{PLA} / \mathrm{CHCl}_{3}$ solutions of $(\mathrm{A}) 0.3 \%\left(\mathrm{Mn}=6.43 \times 10^{4}\right)$, (B) $1.2 \%\left(\mathrm{Mn}=3.11 \times 10^{4}\right)$, and $(\mathrm{C}) 11 \%\left(\mathrm{Mn}=6.29 \times 10^{3}\right)$. TEBAC (5\%) with respect to the polymer used was added to the above polymer solutions. SEM, scanning electron microscopy; PLA, poly(L-lactide); Mn, number average molecular weight; TEBAC, triethyl benzyl ammonium chloride.

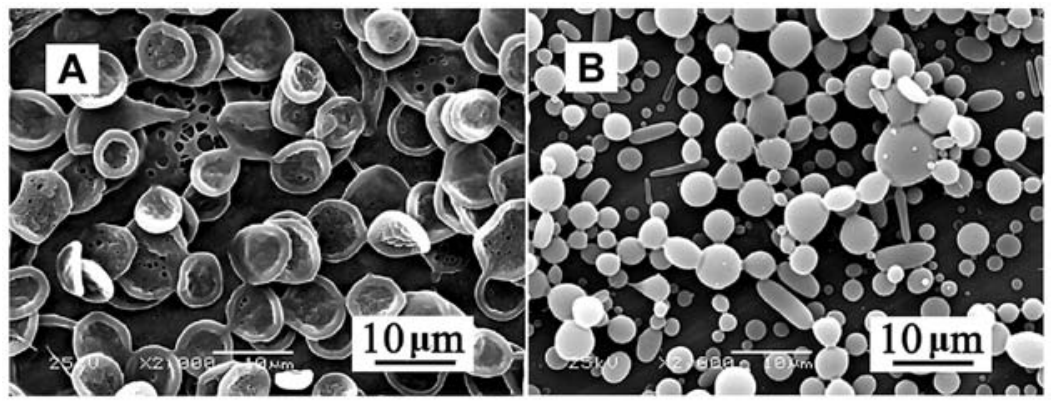

Figure 4. SEM images of electrospraying (A) $0.3 \% \mathrm{PLA}\left(\mathrm{Mn}=6.43 \times 10^{4}\right) / \mathrm{CHCl}_{3}$ solution without the surfactant TEBAC, and (B) $11 \% \mathrm{PLA}\left(\mathrm{Mn}=6.29 \times 10^{3}\right) / \mathrm{CHCl}$ solution containing $8 \%$ TEBAC with respect to the polymer. SEM, scanning electron microscopy; PLA, PLA, poly(L-lactide); Mn, number average molecular weight; TEBAC, triethyl benzyl ammonium chloride.

Drug-loaded PLA microspheres. The rifampin-loaded microspheres presented a brick red color and the pure PLA microspheres presented a white color (Fig. 5A). The FTIR spectra, where the medicated PLA carriers presented the characteristic peaks of both PLA and rifampin, at $1,756 \mathrm{~cm}^{-1}$ and $1,188 \mathrm{~cm}^{-1}$, and $1,526 \mathrm{~cm}^{-1}$ and $1589 \mathrm{~cm}^{-1}$, respectively are shown in Fig. 5B. No new peaks or any shift in the peak sites was observed.

The drug release profiles for the medicated microspheres are presented in Fig. 6. Hardly any release of the drug was observed in the microspheres withoug proteinase $\mathrm{K}$ throughout the 60-h period. However, the amount of drug released increased rapidly with the passage of time when the enzyme was used. No burst release phenomenon was observed, and the release percentage of rifampin was $60 \%$ at $60 \mathrm{~h}$.

\section{Discussion}

Changing the polymer concentration can vary the solution viscosity. The viscoelasticity of the polymer solution, charge 

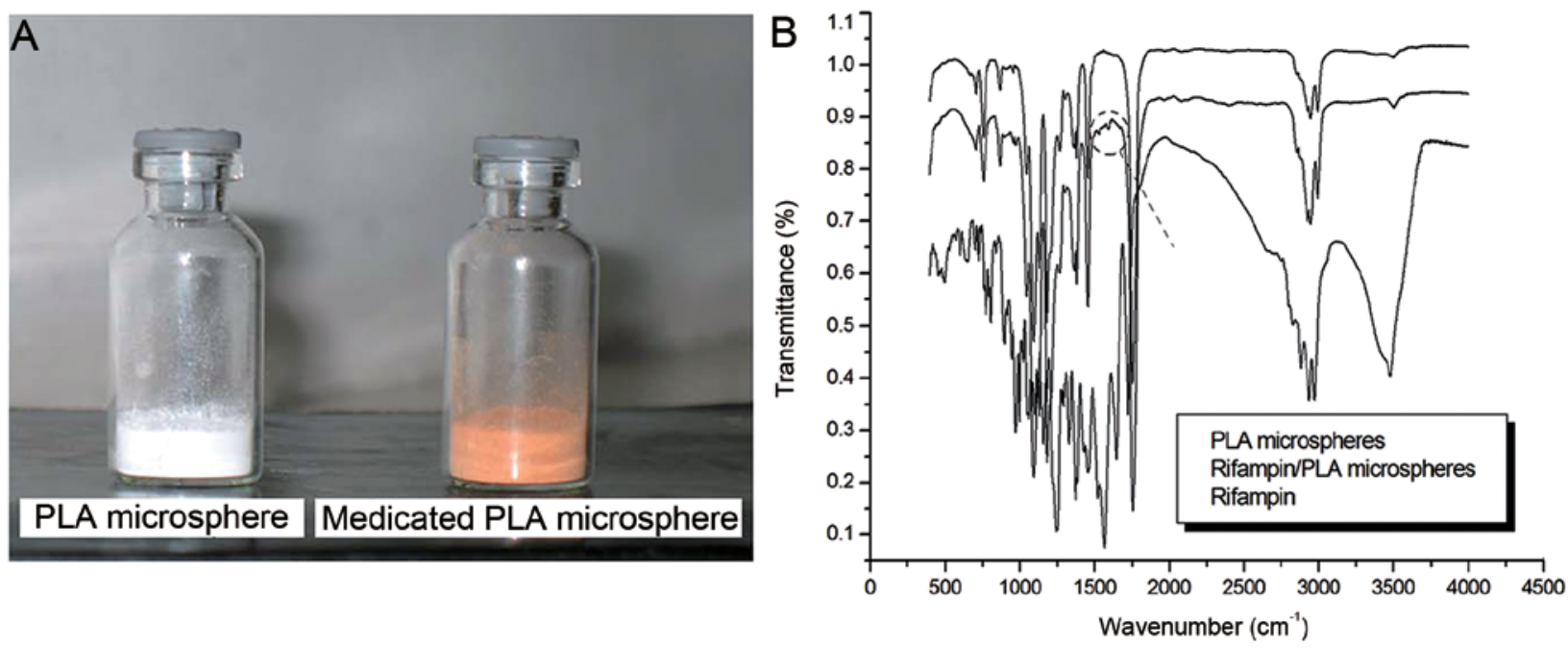

Figure 5. (A) The PLA microshpere and the medicated microsphere obtained by electrospraying. (B) FTIR spectrum of rifampin, PLA microspheres, rifampin/PLA composite microspheres. PLA, PLA, poly(L-lactide); FTIR, Fourier transform infrared spectroscopy.

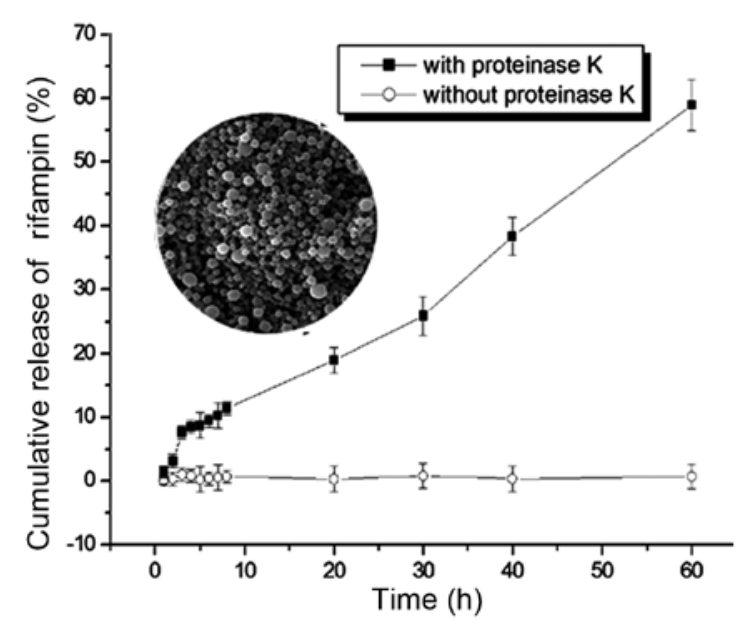

Figure 6. Release percentage of rifampin from electrosprayed microspheres vs. time. The inset shows the SEM image of medicated PLA microshperes obtained by eletrospraying. The molecular weight of PLA is $M n=6.29 \times 10^{3}$ SEM, scanning electron microscopy; PLA, poly(L-lactide); Mn, number average molecular weight.

density on the jet and the surface tension of the solution are the key factors that influence the morphology of eletrosprayed products (28). In case of dilute polymer solutions, the low viscoelastic force of the solution jet is not resistant enough to the stretch exerted by the electrical force, and consequently, the repulsive Coulomb force during the time-of-flight mass spectrum results in the fragmentation of the electrically driven jet. Surface tension controls the contraction of the jet for the formation of beads (29). The separate beads are obtained on the collector. When the concentration of the polymer in the solution is above the non-fiber forming critical concentration (Table I), a thin filament appears between two drops, which, instead of breaking rapidly, assumes the shape of the beads, and changes from a spherical shape to a spindle-like appearance. With the increasing concentration of the polymer solution, the viscoelastic force resists the breaking effect of electrical force and surface tension on the jet, which is reflected as an expulsion of the jet, and the development of filaments along the electrical field.
Another important parameter that influences the morphology of electrospray products is polymeric molecular weight, and hence, we defined the non-fiber forming critical concentration for the polymer at each weight average molecular weight. The results are shown in Table I. The non-fiber forming critical concentration is influenced by the polymer molecular weight, and it decreases with an increase in the polymer molecular weight. When working in the non-fiber concentration range, the polymer solution ejects through the capillaries in the electric field, and within a few milliseconds, countless solution drops are formed. During the flight towards the collector, the solvent evaporates, and the solution concentration rises up, leading to the precipitation of the polymer, and finally, the drops solidify. In the case of high molecular weight polymers, the entanglement between polymer molecules occurs easily due to the longer backbone chain or the more pendant groups, and even at relative low concentration, fibers are obtained (30). Hence, a high molecular weight PLA serves as particle framework, at a lower concentration. Electrospray drops retain a spherical structure due to surface tension during the flight, but at extremely low concentrations, the drops can be hollow. Thereafter, the drops bump at the collector, and their surfaces subside to form hollow semis-pheres.

The surfactant plays a role of solubilization in the process of dissolution (31). For example, benzene is immiscible in water, but its solubility is improved after the addition of sodium oleate into water. Solubilization is different from dissolution. Solubilized benzene is not uniformly dispersed in water, but is rather entrapped in the forming of the oleate micelles. Hence, in order to refine the structure of the product, in this study, varying amounts of surfactant TEBAC were added to dilute the polymer solution. SEM images of electrospraying 0.3\% PLA $\left(\mathrm{Mn}=6.43 \times 10^{4}\right) / \mathrm{CHCl}_{3}$ solution with or without the surfactant, TEBAC are shown in Figs. 3A and 4A. The surfactant plays an important role in influencing the product structure. However, the results obtained in this study were unexpected. The products with TEBAC assumed a hollow semi-spherical structure, and their surfaces were compact and smooth (Fig. 3A), while those without TEBAC presented a cake-like structure with few 


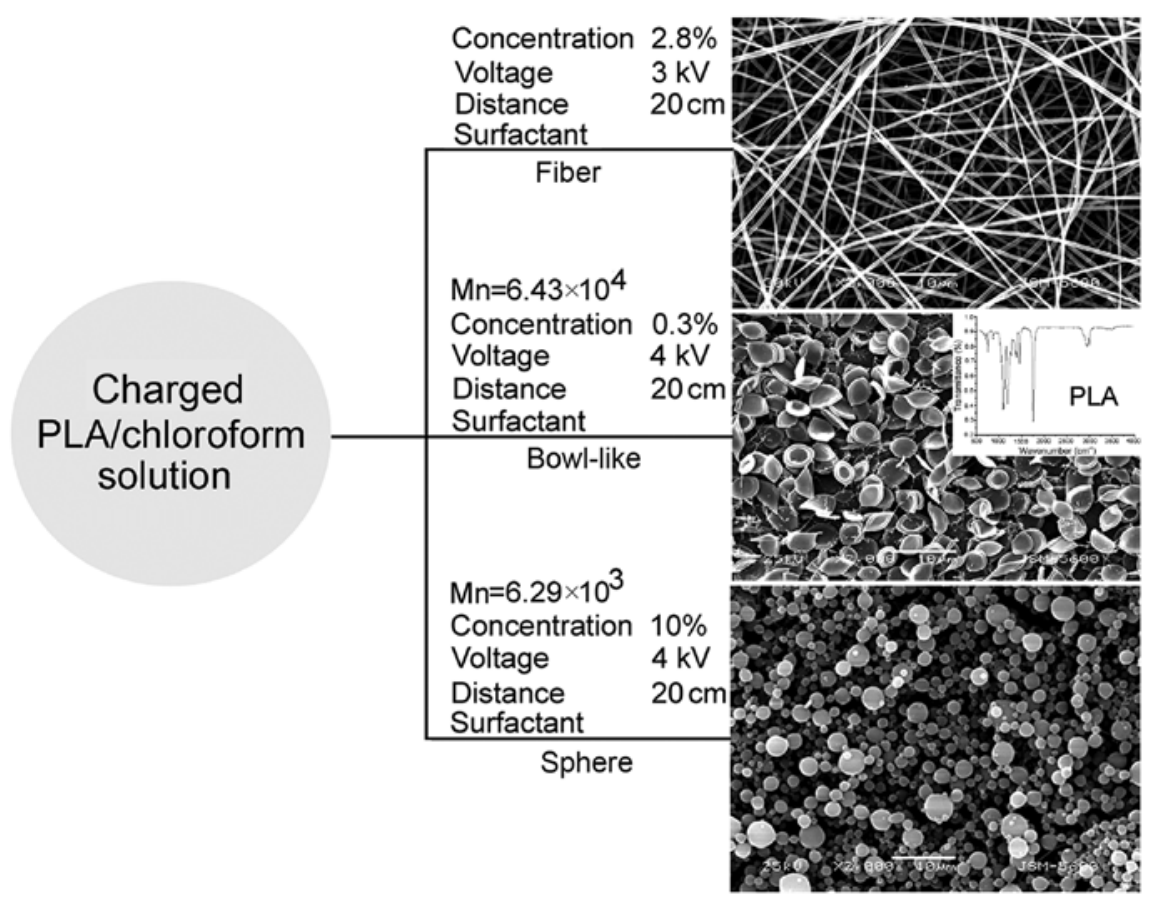

Figure 7. Formation conditions of different morphological PLA microparticles. PLA, poly(L-lactide).

Table I. Polymers and non-fiber forming critical concentrations.

\begin{tabular}{lcccc}
\hline $\begin{array}{l}\text { PLA } \\
\text { samples }\end{array}$ & $\mathrm{Mn}$ & $\mathrm{Mw}$ & $\begin{array}{c}\text { Poly } \\
\text { dispersity }\end{array}$ & $\begin{array}{c}\text { Non-fiber } \\
\text { forming critical } \\
\text { concentration (\%) }\end{array}$ \\
\hline 1 & $6.29 \times 10^{3}$ & $8177 \times 10^{3}$ & 1.3 & $-\mathrm{wt}$ \\
2 & $3.11 \times 10^{4}$ & $7.13 \times 10^{4}$ & 2.2 & $1.2 \mathrm{wt}$ \\
3 & $5.62 \times 10^{4}$ & $7.97 \times 10^{4}$ & 1.4 & $1.0 \mathrm{wt}$ \\
4 & $6.43 \times 10^{4}$ & $1.04 \times 10^{5}$ & 1.6 & $0.5 \mathrm{wt}$ \\
\hline
\end{tabular}

PLA, poly(L-lactide); Mn, number average molecular weight; Mw, weight average molecular weight.

of pores on their surface (Fig. 4A). This may have occurred as the surfactant solubilizes and improves electrical conductivity. Moreover, the effects of varying TEBAC concentrations on electosprayed products are shown in Figs. 3C and 4B. The products synthesized with PLA solution containing 5\% TEBAC had better morphology (Fig. 3C) than those containing $8 \%$ TEBAC (Fig. 4B). Excessive surfactant produces a great deal of conglomeration of the polymer molecule, resulting in conglutination between two particles. Hence, the excess amount of surfactant deteriorated the morphology.

Rifampin and PLA $\left(6.29 \times 10^{3}\right)$ solutions were dissolved and sprayed to obtain the drug microspheres. The preparation of the drug-loaded microspheres through electrospraying is a simple and efficient method, and different from other process, with potential for application on an industrial scale. To ascertain the state of rifampin in the particles, pure rifampin, blank PLA microparticles, and the medicated PLA microcarriers were characterized by FTIR spectroscopy. No new peaks or any shift in the peak sites was observed (Fig. 5B), which suggested that rifampin had physically mixed with PLA. From the inset in
Table II. Results of the stability test.

\begin{tabular}{lcccc}
\hline Time (days) & Color & Weight & Content & $\begin{array}{c}\text { Release } \\
\text { percentage }\end{array}$ \\
\hline 70 & - & - & - & - \\
140 & - & - & + & + \\
\hline
\end{tabular}

The '+' and '-' symbols indicate that there are variations or no variations, respectively following sample degradation.

Fig. 6 it can be seen that rifampin crystals were not detected by optical or electronic microscopy, neither on the surface of the particles nor outside the particles, indicating that rifampin was included within the particles.

Drug release from a biodegradable polymer occurs by diffusion and matrix erosion following degradation. The stability of the medicated microcarriers is shown in Table II. Compared to the initial sample, there was a negligible variation in both weight and the color of the samples. The drug content and release percentage increased slightly at 140 days. This may be due to the degradation of PLA during the test. Nevertheless, the PLGA microspheres were also prepared by the same technique as drug-loaded microspheres.

In this study, PLA microcarriers of varying morphologies were synthesized by refining the eletrospraying process. The results revealed that processing parameters, such as solution concentration, polymer molecular weight, surfactants and solvents play an important role in electrospraying. The association between microcarrier morphology and processing parameters was determined (Fig. 7). The drug-loaded PLA microspheres and PLGA microspheres were also produced as per the above relational model. Drug-loaded microspheres can sustain release in vitro for over $60 \mathrm{~h}$, and the release is stable for 
over 20 weeks. Our study focused on obtaining electrosprayed medicated microparticles from complex polyester particles; further studies are warranted to investigate their commercial use.

\section{Acknowledgements}

The authors gratefully acknowledge the support from the Natural Science Foundation of Guangxi Province (Z2014295).

\section{References}

1. Champion JA, Katare YK and Mitragotri S: Particle shape: a new design parameter for micro- and nanoscale drug delivery carriers. J Control Release 121: 3-9, 2007.

2. Simone EA, Dziubla TD and Muzykantov VR: Polymeric carriers: role of geometry in drug delivery. Expert Opin Drug Deliv 5: 1283-1300, 2008.

3. Yoo JW, Doshi N and Mitragotri S: Adaptive micro and nanoparticles: Temporal control over carrier properties to facilitate drug delivery. Adv Drug Deliv Rev 63: 1247-1256, 2011.

4. Kim DK and Dobson J: Nanomedicine for targeted drug delivery. J Mater Chem 19: 6294-6307, 2009.

5. Venkataraman S, Hedrick JL, Ong ZY, et al: The effects of polymeric nanostructure shape on drug delivery. Adv Drug Deliv Rev 63: 1228-1246, 2011.

6. Geng Y, Dalhaimer P, Cai S, et al: Shape effects of filaments versus spherical particles in flow and drug delivery. Nat Nanotechnol 2: 249-255, 2007.

7. Simone EA, Dziubla TD, Discher DE and Muzykantov VR: Filamentous polymer nanocarriers of tunable stiffness that encapsulate the therapeutic enzyme catalase. Biomacromolecules 10: 1324-1330, 2009.

8. Tayyaba S, Ashraf MW, Nisar A, Afzulpurkar N and Naim Ashraf M: Simulation of dual radii polymeric microneedle array for blood extraction. Emerging Technologies (ICET), 2010 6th International Conference on, pp110-113, 2010.

9. Girones Molera J, Mendez JA and San Roman J: Bioresorbable and nonresorbable polymers for bone tissue engineering. Curr Pharm Des 18: 2536-2557, 2012.

10. Mahmoudi M, Sant S, Wang B, Laurent S and Sen T: Superparamagnetic iron oxide nanoparticles (SPIONs): development, surface modification and applications in chemotherapy. Adv Drug Deliv Rev 63: 24-46, 2011.

11. Farokhzad OC, Radovic-moreno AF and Langer RS: System for Screening Particles. US Patent, 20090298710. Filed December 3, 2009.

12. Sinha R, Kim GJ, Nie S and Shin DM: Nanotechnology in cancer therapeutics: bioconjugated nanoparticles for drug delivery. Mol Cancer Ther 5: 1909-1917, 2006.

13. Takeuchi H, Yamamoto $\mathrm{H}$ and Kawashima Y: Mucoadhesive nanoparticulate systems for peptide drug delivery. Adv Drug Deliv Rev 47: 39-54, 2001.

14. Kim SH, Jeong JH, Chun KW and Park TG: Target-specific cellular uptake of PLGA nanoparticles coated with poly(L-lysine)poly(ethylene glycol)-folate conjugate. Langmuir 21: 8852-8857, 2005.
15. Fahmy TM, Fong PM, Park J, Constable T and Saltzman WM: Nanosystems for simultaneous imaging and drug delivery to T cells. AAPS J 9: E171-E180, 2007.

16. El Badawy AM, Luxton TP, Silva RG, Scheckel KG, Suidan MT and Tolaymat TM: Impact of environmental conditions $(\mathrm{pH}$, ionic strength, and electrolyte type) on the surface charge and aggregation of silver nanoparticles suspensions. Environ Sci Technol 44: 1260-1266, 2010.

17. Zhang W, D'Agosto F, Boyron O, Rieger J and Charleux B: Toward a better understanding of the parameters that lead to the formation of nonspherical polystyrene particles via RAFT-mediated one-pot aqueous emulsion polymerization. Macromolecules 45: 4075-4084, 2012.

18. Hung LH, Teh SY, Jester J and Lee AP: PLGA micro/nanosphere synthesis by droplet microfluidic solvent evaporation and extraction approaches. Lab Chip 10: 1820-1825, 2010.

19. Beck-Broichsitter M, Schweiger C, Schmehl T, Gessler T, Seeger W and Kissel T: Characterization of novel spray-dried polymeric particles for controlled pulmonary drug delivery. J Control Release 158: 329-335, 2012.

20. Nandiyanto ABD and Okuyama K: Progress in developing spraydrying methods for the production of controlled morphology particles: From the nanometer to submicrometer size ranges. Adv Powder Technol 22: 1-19, 2011.

21. Liu W, Wu WD, Selomulya $C$ and Chen XD: Facile spray-drying assembly of uniform microencapsulates with tunable core-shell structures and controlled release properties. Langmuir 27: 12910-12915, 2011.

22. Oh DH, Balakrishnan P, Oh YK, Kim DD, Yong CS and Choi HG: Effect of process parameters on nanoemulsion droplet size and distribution in SPG membrane emulsification. Int J Pharm 404: 191-197, 2011.

23. Liu K, Ding HJ, Liu J, Chen Y and Zhao XZ: Shape-controlled production of biodegradable calcium alginate gel microparticles using a novel microfluidic device. Langmuir 22: 9453-9457, 2006.

24. Cloupeau M and Prunet-Foch B: Electrohydrodynamic spraying functioning modes: a critical review. J Aerosol Sci 25: 1021-1036, 1994.

25. Park S, Kim JB, Lee S, Bang JH and Kim YS: Synthesis of titanium oxide nanocups by electrospraying. Mat Lett 69: 34-36, 2012.

26. Rietveld IB, Kobayashi K, Yamada H and Matsushige K: Morphology control of poly(vinylidene fluoride) thin film made with electrospray. J Colloid Interface Sci 298: 639-651, 2006.

27. Sun L, Yu X, Sun M, et al: Preparation of quantum dots encoded microspheres by electrospray for the detection of biomolecules. J Colloid Interface Sci 358: 73-80, 2011.

28. Luo CJ, Stride E and Edirisinghe M: Mapping the influence of solubility and dielectric constant on electrospinning polycaprolactone solutions. Macromolecules 45: 4669-4680, 2012.

29. Magarvey RH and Outhouse LE: Note on the break-up of a charged liquid jet. J Fluid Mech 13: 151-157, 1962.

30. Renekern DH and Chun I: Nanometre diameter fibres of polymer, produced by electrospinning. Nanotechnology 7: 216-223, 1996.

31. Taylor DJ, Thomas RK and Penfold J: Polymer/surfactant interactions at the air/water interface. Adv Colloid Interface Sci 132. 69-110, 2007 